\title{
Amphipleuraceae e Diploneidaceae (Bacillariophyceae) da bacia do rio Iguaçu, PR, Brasil
}

\author{
Nicole M. Brassac ${ }^{1,3}$ e Thelma A. Veiga Ludwig²
}

Recebido em 03/07/2003. Aceito em 08/10/2004

\begin{abstract}
RESUMO - (Amphipleuraceae e Diploneidaceae (Bacillariophyceae) da bacia do rio Iguaçu, PR, Brasil). O presente trabalho é uma contribuição ao levantamento florístico das diatomáceas dos rios da área de abrangência do reservatório da Usina Hidrelétrica de Salto Caxias, localizada no Município de Capitão Leônidas Marques, sudoeste do Estado do Paraná, Brasil. Coletas foram realizadas, mensalmente, de março/1997 a fevereiro/1998, em oito estações de amostragem abrangendo o rio Iguaçu e seus afluentes: os rios Cotegipe, Jaracatiá, Chopim, Tormenta, Adelaide e Guarani. Cinco táxons pertencentes à família Amphipleuraceae e quatro pertencentes à família Diploneidaceae foram registrados, sendo que Frustulia vitrea Oestrup e Diploneis ovalis (Hilse) Cleve foram registros pioneiros para o Estado do Paraná.
\end{abstract}

Palavras-chave: Amphipleuraceae, Diploneidaceae, diatomáceas, bacia hidrográfica do Iguaçu, reservatório

\begin{abstract}
Amphipleuraceae and Diploneidaceae (Bacillariophyceae) from Iguaçu basin river, PR, Brazil). The survey is a floristic inventory of Amphipleuraceae and Diploneidadaceae from rivers of the region to be flooded for the construction of Caxias power plant, city of Capitão Leônidas Marques, southwest of Paraná State, Brazil. Samples were collected from March, 1997 to February, 1998 , in the Iguaçu river and its tributaries: Cotegipe, Jaracatiá, Chopim, Tormenta, Adelaide and Guarani rivers. Amphipleuraceae family was represented by five infraspecific taxa and the Diploneidaceae by four. Frustulia vitrea Oestrup and Diploneis ovalis (Hilse) Cleve were registered for the first time to Paraná State.
\end{abstract}

Key words: Amphipleuraceae, Diploneidaceae, diatoms, Iguaçu basin, reservoir

\section{Introdução}

A flora diatomológica de ecossistemas lóticos continentais brasileiros tem sido pouco estudada, considerando a extensão da rede hidrográfica do país. Grande parte das contribuições resulta de trabalhos desenvolvidos nos Estados do sul e sudeste do Brasil, principalmente, Paraná e Rio Grande do Sul. É oportuno ressaltar que dissertações de mestrado concentram a maior parte das informações reunidas e muitas não estão publicadas, constituindo-se em literatura de difícil acesso para a maioria dos pesquisadores.

Ludwig \& Flôres $(1995 ; 1997)$ iniciaram o estudo da diatomoflórula de ambientes lóticos paranaenses em rios a serem represados para a construção da usina hidrelétrica de Segredo, na bacia do rio Iguaçu. As classes abordadas foram Coscinodiscophyceae, Bacillariophyceae e Fragilariophyceae. Contin (1990) realizou amostragem na região da barragem de captação d'água do rio Iguaçu, em Curitiba e Brassac et al. (1999) identificaram as diatomáceas cêntricas de rios da região de abrangência da usina hidrelétrica de Salto Caxias. Bittencourt-Oliveira (2002), em trabalho sobre a comunidade fitoplanctônica do rio Tibagi, registrou 52 diatomáceas. Espécies perifíticas de Navicula foram estudadas por Leandrini et al. (2002), em amostras do rio Pirapó e córrego Sarandi, na região de Maringá.

A família Amphipleuraceae Grunow inclui quatro gêneros, de acordo com Round et al. (1990): Frickea, Amphipleura, Frustulia e Cistula. Destes, apenas Amphipleura e Frustulia foram encontrados na área de estudo. Round et al. (1990) comentaram que ambos os gêneros estão intimamente relacionados e, segundo Patrick \& Reimer (1966), diferenciam-se entre si apenas pela estrutura da rafe e da área central. A família Diploneidaceae Mann inclui dois gêneros, Diploneis e Raphidodiscus. O gênero Diploneis, registrado no presente trabalho, é bastante característico pela presença de canais longitudinais em

1 Núcleo de Ciências Biológicas e da Saúde, Centro Universitário Positivo, UnicenP, Rua Prof. Pedro Viriato Parigot de Souza, 5300, Ecoville, CEP 81280-330, Curitiba, PR, Brasil

2 Universidade Federal do Paraná, Departamento de Botânica, Centro Politécnico, C. Postal 19031, CEP 81530-990, Curitiba, PR, Brasil

3 Autor para correspondência: nicole@unicenp.br 
ambos os lados da rafe e ornamentações grosseiras na superfície valvar, sob microscopia óptica (Idei \& Kobayasi 1989; Round et al. 1990).

Objetivou-se no presente trabalho, dar continuidade ao inventário florístico das diatomáceas pertencentes às famílias Amphipleuraceae e Diploneidaceae de ecossistemas lóticos do Estado do Paraná, registrar a variabilidade morfológica dos mesmos, discutir a problemática taxonômica e contribuir para o conhecimento e registro geográfico da diatomoflórula paranaense.

\section{Material e métodos}

A bacia hidrográfica do rio Iguaçu é o maior complexo hídrico do Estado do Paraná, ocupando área de $55.024 \mathrm{~km}^{2}$ (Suderhsa 1995). A usina hidrelétrica de Salto Caxias, área de propriedade da Companhia Paranaense de Energia Elétrica (COPEL), opera no rio Iguaçu, município de Capitão Leônidas Marques, a sudoeste do Estado (Intertechne 1993). O estudo foi realizado em ambientes lóticos situados na área de influência do reservatório da usina, entre as

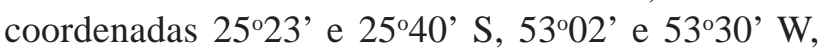
abrangendo cinco municípios paranaenses. Selecionaram-se oito estações de coleta, localizadas no rio Iguaçu e afluentes: rios Cotegipe, Adelaide, Jaracatiá, Tormenta, Guarani e Chopim (Tab. 1).

Coletas mensais foram realizadas pela Equipe de campo da COPEL, durante 12 meses, de março/1997 a fevereiro/1998, com rede de fitoplâncton (abertura de malha de $25 \mu \mathrm{m}$ ), e fixadas com solução aquosa de formol a $4 \% \mathrm{v} / \mathrm{v}$. Amostras líquidas encontram-se tombadas no Herbário da Universidade Federal do Paraná (UPCB 34879 a 35047). Lâminas permanentes foram utilizadas para o estudo qualitativo das diatomáceas, uma série confeccionada com material não

Tabela 1. Estações de coleta no rio Iguaçu e seus afluentes, PR, Brasil.

\begin{tabular}{|c|c|c|}
\hline Estações & Rios & Municípios \\
\hline 1 & Iguaçu & $\begin{array}{l}\text { Capitão Leônidas Marques, próximo } \\
\text { ao eixo da barragem }\end{array}$ \\
\hline 2 & Cotegipe & Nova Prata do Iguaçu \\
\hline 3 & Adelaide & Três Barras do Paraná \\
\hline 4 & Tormenta & Três Barras do Paraná \\
\hline 5 & Guarani & $\begin{array}{l}\text { Três Barras do Paraná, próximo à } \\
\text { reserva do Guarani }\end{array}$ \\
\hline 6 & Jaracatiá & Salto do Lontra \\
\hline 7 & Chopim & Barra do Chopim \\
\hline 8 & Iguaçu & Barra do Chopim \\
\hline
\end{tabular}

oxidado e outra com amostras submetidas à oxidação (Moreira Filho \& Valente-Moreira 1981), utilizando-se Naphrax como meio de inclusão. As ilustrações foram obtidas com filmes Imagelink HQ (Kodak). O sistema de classificação utilizado foi o proposto por Round et al. (1990).

\section{Resultados e discussão}

\section{Amphipleura Kützing}

Amphipleura lindheimerii Grunow var. lindheimerii, Verh. Zool. -Bot. Ges. Wein 12: 469, pl. 13, fig. 11a-b. 1862.

Fig. 1.

Valvas rombo-lanceoladas a lanceoladas; extremidades atenuado-arredondadas; área axial linear, estreita, alargando-se nas porções terminais da valva; área central ausente; ramos da rafe incluídos em costela, medindo $1 / 5$ a $1 / 6$ compr. valvar; estrias transapicais paralelas e longitudinais levemente onduladas, de difícil visualização. Eixo apical: 120 a $196 \mu$ m; eixo transapical: 13,6 a $34 \mu \mathrm{m}$.

Distribuição no Estado do Paraná: Curitiba (Cecy 1986; Contin 1990; Lozovei \& Shirata 1990); Maringá (Rodrigues 1991); Ponta Grossa (Moro \& Fürstenberger 1993); Londrina (Bittencourt-Oliveira 2002).

Material examinado: BRASIL. Paraná: Capitão Leônidas Marques, rio Iguaçu, 2/IV/1997, Souza et al. s.n. (UPCB 34893); 5/V/1997, (UPCB 34906); 2/VI/1998, (UPCB 34919); 1/VII/1997, (UPCB 34932); 11/VII/1997, (UPCB 34947); 2/IX/1997, (UPCB 34960); 3/XI/1997, (UPCB 34990); 1/XII/1997, (UPCB 35005); 7/I/1998, (UPCB 35020); 3/II/1998, (UPCB 35035); Nova Prata do Iguaçu, rio Cotegipe, 3/III/1997, Souza et al. s.n. (UPCB 34880); 2/IV/1997, (UPCB 34894); 5/V/1997, (UPCB 34907); 2/VI/1998, (UPCB 34920); 1/VII/1997, (UPCB 34933); 2/IX/1997, (UPCB 34961); 1/XII/1997, (UPCB 35006); 7/I/1998, (UPCB 35021) 3/II/1998, (UPCB 35036); Três Barras do Paraná, rio Adelaide, 5/III/1997, Souza et al. s.n. (UPCB 34882); 5/IV/1997, (UPCB 34896); 8/V/1997, (UPCB 34909); 5/VI/1997, (UPCB 34922); 4/VII/1997, (UPCB 34935); 5/VII/1997, (UPCB 34950); 5/IX/1997, (UPCB 34963); 4/X/1997, (UPCB 34978); 6/XI/1997, (UPCB 34993); 4/XII/1997, (UPCB 35008); 10/I/1998, (UPCB 35023); Três Barras do Paraná, rio Tormenta, 6/III/1997, Souza et al. s.n. (UPCB 34884); 2/IV/1997, (UPCB 34898); 5/V/1997, (UPCB 34911); 2/VI/1997, (UPCB 34924); 1/VII/1997, (UPCB 34937); 
11/VII/1997, (UPCB 34948); 11/VII/1997, (UPCB 34952); 5/XI/1997, (UPCB 34995); 7/I/1998, (UPCB 35025); 3/II/1998, (UPCB 35040); Três Barras do Paraná, rio Guarani, 9/III/1997, Souza et al. s.n. (UPCB 34887); 7/IV/1997, (UPCB 34901); 10/V/1997, (UPCB 34914); 5/VI/1997, (UPCB 34927); 6/VII/1997, (UPCB 34940); 5/VII/1997, (UPCB 34955); 7/IX/1997, (UPCB 34968); 6/X/1997, (UPCB 34983); 6/XII/1997, (UPCB 35013); 12/I/1998, (UPCB 35028); 8/II/1998, (UPCB 35043); Salto do Lontra, rio Jaracatiá, 11/III/1997, Souza et al. s.n. (UPCB 34889); 8/IV/1997, (UPCB 34903); 11/V/1997, (UPCB 34916); 8/VI/1997, (UPCB 34929); 7/VII/1997, (UPCB 34942); 8/VII/1997, (UPCB 34957); 8/IX/1997, (UPCB 34970);
7/X/1997, (UPCB 34985); 9/XI/1997, (UPCB 35000); 7/XII/1997, (UPCB 35015); 13/I/1998, (UPCB 35030); 9/II/1998, (UPCB 35045); Barra do Chopim, rio Chopim, 12/III/1997, Souza et al. s.n. (UPCB 34890); 8/VI/1997, (UPCB 34930); 7/VII/1997, (UPCB 34943); 8/IX/1997, (UPCB 34971); 9/XI/1997, (UPCB 35001); 7/XII/1997, (UPCB 35016); 7/X/1997, (UPCB 34986); 13/I/1998, (UPCB 35031); 9/II/1998, (UPCB 35046); Barra do Chopim, rio Iguaçu, 12/III/1997, Souza et al. s.n. (UPCB 34891); 10/IV/1997, (UPCB 34905); (UPCB 34931); 9/VII/1997, (UPCB 34959); 10/IX/1997, (UPCB 34972); 11/XI/1997, (UPCB 35002); 9/XII/1997, (UPCB 35017); 15/I/1998, (UPCB 35032); 11/II/1998, (UPCB 35047).

Frustulia Rabenhorst

Chave para os táxons de Frustulia

1. Rafe arqueada 4. F. vulgaris var. vulgaris

1. Rafe reta

2. Extremidades atenuado-arredondadas 3. F. vitrea var. vitrea

2. Extremidades rostradas a subcapitadas

3. Valvas rombo-lanceoladas, margens onduladas 1. F. crassinervia var. crassinervia

3. Valvas linear-lanceoladas, margens não onduladas 2. F. saxonica var. saxonica

1. Frustulia crassinervia (Brébisson) Costa var. crassinervia, Diat. Res. Biol. Poço das Antas, RJ, Brasil, Iheringia: ser. Bot. 46:57-143. 1995

Navicula crassinervia Brébisson ex Wm. Smith, Syn. British Diat., 1(47), pl. 31, fig. 271. 1853.

Fig. 2-6.

Valvas rombo-lanceoladas a rômbicas com margens onduladas; extremidades rostradas a subcapitadas; área axial linear, estreita; área central reduzida e elíptica; rafe reta situada entre costelas lineares; estrias geralmente conspícuas, transversais paralelas ao longo da valva a levemente convergentes nas extremidades e longitudinais onduladas. Eixo apical: 37,6 a $64 \mu \mathrm{m}$; eixo transapical: 9,6 a $14 \mu \mathrm{m}$; estrias transapicais: 26 a 32 em $10 \mu \mathrm{m}$; estrias longitudinais: 26 a 32 em $10 \mu \mathrm{m}$.

Patrick \& Reimer (1966) afirmaram que existe grande confusão na literatura na distinção entre a F. crassinervia e $F$. saxonica e optaram por separá-las em função da visível diferença no contorno valvar, com margens fortemente onduladas na prim,eira espécie e lisas na segunda. Também, as extremidades de F. saxonica são abruptamente atenuado-rostradas e em $F$. crassinervia, as margens começam a se estreitar desde a região mediana valvar, com extremidades subcapitadas a rostradas.

Outros táxons apresentam semelhança com F. crassinervia, tal como F. undosa descrita por Metzeltin \& Lange-Bertalot (1998) a partir de amostras venezuelanas. Segundo os autores, F. undosa caracteriza-se por valvas mais estreitas, o que resulta numa relação comprimento/largura proporcionalmente maior do que em $F$. crassinervia. Além disto, as ondulações das margens valvares são mais pronunciadas nos terços distais e as estrias são paralelas na porção mediana a fortemente convergentes nas extremidades. F. neocaledonica Manguin é outro táxon apresentando afinidades morfológicas com F. crassinervia; mas segundo Moser et al. (1995), diferencia-se da última por apresentar uma valvocópula bastante característica, como apresentada na prancha 41, figuras 5 e 6 da obra acima citada.

Moser et al. (1995) e Metzeltin \& Lange-Bertalot (1998) registraram vários exemplares do gênero Frustulia com margens onduladas, pertencentes a espécies e variedades distintas. Enquanto na primeira obra os táxons mostram-se com circunscrições definidas, na segunda, as características diagnósticas 
não estão bem elucidadas, dificultando a delimitação entre os táxons propostos e tornando a característica ondulada das margens sem consistência taxonômica.

Portanto, propõem-se que, juntamente com a característica ondulada das margens, outros fatores sejam considerados em conjunto para a identificação de táxons infragenéricos de Frustulia, tais como: o padrão de estriação, a silicificação da frústula e a morfologia das extremidades e do contorno valvar.

De acordo com o que foi observado dentro da população ocorrente na área da Usina, a ondulação das margens, as estrias bastante conspícuas e a estrutura valvar fortemente silicificada delimitam melhor F. crassinervia. Sugerem-se estudos mais profundos sob microscopia eletrônica de varredura para esclarecer as dúvidas taxonômicas. Seria interessante dar enfoque à estrutura da valvocópula, que parece ser uma característica de peso taxonômico para a identificação de algumas espécies do gênero (Moser et al. 1995).

Distribuição no Estado do Paraná: Cascavel (Tavares \& Valente-Moreira 2000).

Material examinado: BRASIL. Paraná: Capitão Leônidas Marques, rio Iguaçu, 3/III/1997, Souza et al. s.n. (UPCB 34879); 5/V/1997, (UPCB 34906); 2/VI/1998, (UPCB 34919); 2/IX/1997, (UPCB 34960); 3/II/1998, (UPCB 35035) Nova Prata do Iguaçu, rio Cotegipe, 7/I/1998, Souza et al. s.n. (UPCB 35021); Três Barras do Paraná, rio Adelaide, 5/IX/1997, Souza et al. s.n. (UPCB 34963); 6/XI/1997, (UPCB 34993); 4/XII/1997, (UPCB 35008); 7/X/1997, (UPCB 34986); Três Barras do Paraná, rio Tormenta, 6/III/1997, Souza et al. s.n. (UPCB 34884); 2/IV/1997, (UPCB 34898); 1/VII/1997, (UPCB 34937); 7/I/1998, (UPCB 35025); 3/II/1998, (UPCB 35040); Três Barras do Paraná, rio Guarani, 6/VII/1997, Souza et al. s.n. (UPCB 34940); 6/X/1997, (UPCB 34983); Barra do Chopim, rio Chopim, 11/V/1997, Souza et al. s.n. (UPCB 34917); 7/VIII/1997, (UPCB 34943); 8/VII/1997, (UPCB 34958); 9/XI/1997, (UPCB 35001); 9/II/1998, (UPCB 35046); Barra do Chopim, rio Iguaçu, 10/IV/1997, Souza et al. s.n. (UPCB 34905); 10/VI/1997, (UPCB 34931); 9/VII/1997, (UPCB 34944); 9/X/1997, (UPCB 34987); 11/II/1998, (UPCB 35047).

2. Frustulia saxonica Rabenhorst var. saxonica, Süssw. -Diat. Freund Mikrosk. p. 50, pl. 7, fig. 1. 1853.

Fig. 7-8.

Valvas linear-lanceoladas a lanceoladas; extremidades rostradas a subcapitadas; área axial linear, estreita; área central constrita; rafe reta situada entre costelas lineares; estrias geralmente conspícuas, transversais paralelas a levemente convergentes nas extremidades e longitudinais onduladas. Eixo apical: 35,2 a 62,4 $\mu$ m; eixo transapical: 9,6 a 14,4 $\mu \mathrm{m}$; 20 a 26 estrias transapicais em $10 \mu \mathrm{m} ; 20$ a 22 estrias longitudinais em $10 \mu \mathrm{m}$.

Distribuição no Estado do Paraná: Curitiba (Contin 1990; Lozovei \& Shirata 1990); Maringá (Rodrigues 1991); Palmeira (Valente-Moreira 1975).

Material examinado: BRASIL. Paraná: Capitão Leônidas Marques, rio Iguaçu, 2/VI/1998, Souza et al. s.n. (UPCB 34919); 1/VII/1997, (UPCB 34932); 2/IX/1997, (UPCB 34960); 1/X/1997, (UPCB 34975); 3/XI/1997, (UPCB 34990); 1/XII/1997, (UPCB 35005); 3/II/1998, (UPCB 35035); Nova Prata do Iguaçu, rio Cotegipe, 1/VII/1997, Souza et al. s.n. (UPCB 34933); 11/VII/1997, (UPCB 34948); 3/XI/1997, (UPCB 34991); 7/I/1998, (UPCB 35021); Três Barras do Paraná, rio Adelaide, 5/III/1997 Souza et al. s.n. (UPCB 34882); 5/VI/1997, (UPCB 34922); 4/VII/1997, (UPCB 34935); 5/VII/1997, (UPCB 34950); 5/IX/1997, (UPCB 34963); 4/X/1997, (UPCB 34978); 6/XI/1997, (UPCB 34993); 10/I/1998, (UPCB 35023); 6/II/1998, (UPCB 35038); Três Barras do Paraná, rio Tormenta, 2/IV/1997, Souza et al. s.n. (UPCB 34898); 2/VI/1997, (UPCB 34924); 5/XI/1997, (UPCB 34995); 11/VII/1997, (UPCB 34952); 1/X/1997, (UPCB 34980); 1/XII/1997, (UPCB 35010); 7/I/1998, (UPCB 35025); 3/II/1998, (UPCB 35040); Três Barras do Paraná, rio Guarani, 7/IX/1997, Souza et al. s.n. (UPCB 34968); 8/II/1998, (UPCB 35043); Salto do Lontra, rio Jaracatiá, 7/VII/1997, Souza et al. s.n. (UPCB 34942); 8/VII/1997, (UPCB 34957); 9/XI/1997, (UPCB 35000); 7/XII/1997, (UPCB 35015); 9/II/1998, (UPCB 35045); Barra do Chopim, rio Chopim, 12/III/1997, (UPCB 34890); 8/IV/1997, (UPCB 34904); 8/VII/1997, (UPCB 34958); 8/IX/1997, (UPCB 34971); 9/XI/1997, 7/X/1997, (UPCB 34986); (UPCB 35001); 7/XII/1997, (UPCB 35016); 13/I/1998, (UPCB 35031); 9/II/1998, (UPCB 35046); Barra do Chopim, rio Iguaçu, 12/III/1997, (UPCB 34891); 9/VII/1997, (UPCB 34944); 9/VII/1997, (UPCB 34959); 10/IX/1997, (UPCB 34972); 9/X/1997, (UPCB 34987); 11/XI/1997, (UPCB 35002); 11/II/1998, (UPCB 35047).

3. Frustulia vitrea Oestrup var. vitrea, Bot. Faeröes (1): 262, fig. 30. 1901.

Fig. 10.

Valvas lanceoladas; extremidades atenuadoarredondadas; área axial linear, estreita; área central 
reduzida e circular; rafe reta situada entre costelas lineares; estrias inconspícuas. Eixo apical: 15,2 a $24 \mu \mathrm{m}$; eixo transapical: 4 a 5,6 $\mu \mathrm{m}$; estrias inconspícuas.

Segundo Cleve-Euler (1952), este táxon caracteriza-se por apresentar valvas linear-lanceoladas até rombo-lanceoladas, nódulo central presente e reduzido, estrias delicadas, aproximadamente $30 \mathrm{em}$ $10 \mu \mathrm{m}$, e geralmente inconspícuas. No material encontrado na área de estudo as estrias apresentaram-se sempre inconspícuas.

Krammer \& Lange-Bertalot (1986) sinonimizaram F. vitrea Oestrup em Navicula festiva Krasske, a qual se caracteriza por apresentar estrias transversais distintas, interrompidas por linha hialina longitudinal e rafe não inserida em costela. É importante ressaltar que Krammer \& Lange-Bertalot (1986) não mencionaram ter observado o material tipo de Oestrup para a sinonimização. Cleve-Euler (1952) já indicava que Navicula vitrea (Oestrup) Hustedt citada e ilustrada em Hustedt (1930) não estava relacionada com Frustulia, mas sim com Navicula festiva Krasske.

Analisando os materiais ilustrados e descritos pelos autores supracitados, sugere-se desconsiderar a sinonimização de Frustulia vitrea em Navicula festiva, pois em nenhum material desta Navicula existe a estrutura típica do gênero Frustulia, ou seja, a rafe incluída em costela longitudinal.

Distribuição no Estado do Paraná: primeira citação de ocorrência do táxon.

Material examinado: BRASIL. Paraná: Capitão Leônidas Marques, rio Iguaçu, 1/X/1997, Souza et al. s.n. (UPCB 34975); Nova Prata do Iguaçu, rio Cotegipe, 11/VII/1997, Souza et al. s.n. (UPCB 34948); 3/XI/1997, (UPCB 34991) 1/X/1997, (UPCB 34976); 7/I/1998, (UPCB 35021); Três Barras do Paraná, rio Adelaide, 4/X/1997, Souza et al. s.n. (UPCB 34978); 10/I/1998, (UPCB 35023); Três Barras do Paraná, rio Tormenta, 1/X/1997, Souza et al. s.n. (UPCB 34980); 1/XII/1997, (UPCB 35010).

4. Frustulia vulgaris (Thwaites) De Toni var. vulgaris, Syn. Alg. (2)1: 280. 1891.

Schizonema vulgare Thwaites, Ann. Mag. Nat. Hist. 2(1): 170, pl. 11, fig. 1-5. 1848.

Fig. 9.

Valvas linear-lanceoladas; extremidades largamente rostradas; área axial linear, estreita; área central elíptica; rafe arqueada situada entre costelas levemente convexas; estrias geralmente conspícuas, porém de difícil contagem, transversais radiadas na porção central. Eixo apical: 36 a $50 \mu$ m; eixo transapical: 8 a $10 \mu \mathrm{m}$; estrias de difícil contagem, aproximadamente 36 em $10 \mu \mathrm{m}$.

Segundo Krammer \& Lange-Bertalot (1986), Frustulia vulgaris difere da maior parte das outras espécies e variedades incluídas no gênero por possuir um nódulo central oval. De acordo com estes autores, o táxon em questão distingue-se de Frustulia rhomboides var. viridula (Brébisson) Cleve no comprimento (80 a $110 \mu \mathrm{m})$ e no contorno da valva (lanceolado) e de formas não capitadas de F. spicula Amossé, pela estrutura do nódulo central, linear, não expandido, na última. Também, F. weinholdii Hustedt é semelhante à $F$. vulgaris, no entanto, Patrick \& Reimer (1966) indicaram que a primeira é característica por apresentar extremidades proximais da rafe fletidas para o mesmo lado, envolvidas por uma ampla área central assimétrica.

Distribuição no Estado do Paraná: Curitiba (Contin 1990; Lozovei \& Shirata 1990); Maringá (Rodrigues 1991).

Material examinado: BRASIL. Paraná: Capitão Leônidas Marques, rio Iguaçu, 2/IV/1997, Souza et al. s.n. (UPCB 34893); 2/IX/1997, (UPCB 34960); 1/X/1997, (UPCB 34975); 3/XI/1997, (UPCB 34990); 1/XII/1997, (UPCB 35005); Nova Prata do Iguaçu, rio Cotegipe, 2/VI/1998, Souza et al. s.n. (UPCB 34920); 2/IX/1997, (UPCB 34961); 1/X/1997, (UPCB 34976); 7/I/1998, (UPCB 35021); 3/II/1998, (UPCB 35036); Três Barras do Paraná, rio Adelaide, 5/IV/1997, Souza et al. s.n. (UPCB 34896); 5/VI/1997, (UPCB 34922); 5/IX/1997, (UPCB 34963); 4/X/1997, (UPCB 34978); 6/XI/1997, (UPCB 34993); 4/XII/1997, (UPCB 35008); 6/II/1998, (UPCB 35038); Três Barras do Paraná, rio Tormenta, 2/VI/1997, Souza et al. s.n. (UPCB 34924); 1/VII/1997, (UPCB 34937); 1/X/1997, (UPCB 34980); 5/XI/1997, (UPCB 34995); 7/I/1998, (UPCB 35025); 3/II/1998, (UPCB 35040); Três Barras do Paraná, rio Guarani, 10/V/1997, Souza et al. s.n. (UPCB 34914); 6/VII/1997, (UPCB 34940); 7/IX/1997, (UPCB 34968); 6/X/1997, (UPCB 34983); Salto do Lontra, rio Jaracatiá, 7/VII/1997, Souza et al. s.n. (UPCB 34942); 8/VII/1997, (UPCB 34957); 7/X/1997, (UPCB 34985); 9/XI/1997, (UPCB 35000); Barra do Chopim, rio Chopim, 7/VII/1997, Souza et al. s.n. (UPCB 34943); 8/IX/1997, (UPCB 34971); 7/X/1997, (UPCB 34986); 13/I/1998, (UPCB 35031); Barra do Chopim, rio Iguaçu, 12/III/1997, Souza et al. s.n. (UPCB 34891); 10/VI/1997, (UPCB 34931); 10/IX/1997, (UPCB 34972); 9/XII/1997, (UPCB 35017); 11/II/1998, (UPCB 35047). 

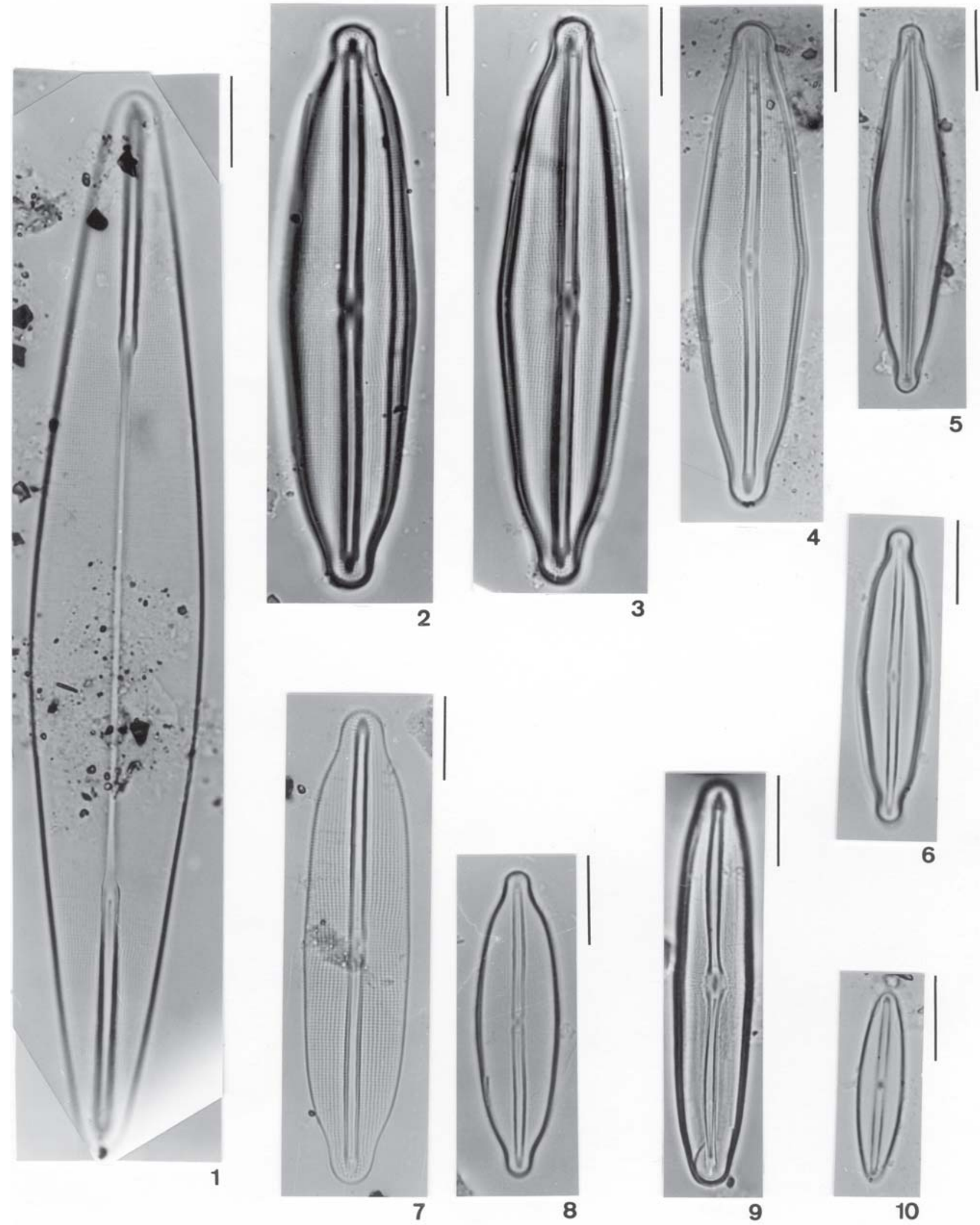

Figuras 1-10. Amphipleuraceae da bacia do rio Iguaçu, PR, Brasil. 1. Amphipleura lindheimerii Grunow var. lindheimerii. 2-6. Frustulia crassinervia (Brébisson) Costa var. crassinervia. 7-8. Frustulia saxonica Rabenhorst var. saxonica. 9. Frustulia vulgaris (Thwaites) De Toni var. vulgaris. 10. Frustulia vitrea Oestrup var. vitrea. 
Diploneis C.G. Ehrenberg

Chave para os táxons de Diploneis

1. Fileira única de aréolas entre cada duas costelas transapicais

1. D. ovalis var. ovalis

1. Fileira dupla de aréolas entre cada duas costelas transapicais

2. Aréolas dispostas em qüincunce

3. D. subovalis var. subovalis

2. Aréolas paralelas entre si

3. Canal ornamentado, longitudinalmente, por fileira única de aréola

2. D. pseudovalis var. pseudovalis

3. Canal ornamentado, longitudinalmente, por mais de uma fileira de aréolas 4. Diploneis sp.

1. Diploneis ovalis (Hilse) Cleve var. ovalis, Diat. Finl. (44), pl. 2, fig. 13. 1891.

Pinnularia ovalis Hilse, in Rabenhorst, Alg. Eur. (1025). 1861.

Fig. 17-18.

Valvas elípticas; extremidades arredondadas; área axial estreita; área central circular; rafe reta incluída em canal longitudinal cuja areolação é distinta do restante da valva, uma aréola presente na continuidade de cada estria; estrias radiadas; formadas por fileira única de aréolas. Eixo apical: 19,2 a 47,2 $\mu$ m; eixo transapical: 9,6 a 19,2 $\mu \mathrm{m} ; 9$ a 15 estrias em $10 \mu \mathrm{m}$.

Segundo Jensen (1985) e Krammer \& LangeBertalot (1986), formas menores de D. ovalis Cleve são facilmente confundidas com $D$. parma Cleve, por causa da sobreposição das medidas e pela presença de uma única fileira de aréolas entre as costelas. Krammer \& Lange-Bertalot (1986) sugeriram análises morfológicas conjuntas nestas populações, bem como em Diploneis subovalis Cleve, para que sejam elucidadas as suas diferenças.

O material examinado apresentou uma ampla variação métrica concordando com as descrições e ilustrações apresentadas por Van Heurck (1880-85), Frenguelli (1923) e Krammer \& Lange-Bertalot (1986).

Distribuição no Estado do Paraná: primeira citação de ocorrência do táxon.

Material examinado: BRASIL. Paraná: Nova Prata do Iguaçu, rio Cotegipe, 3/XI/1997, Souza et al. s.n. (UPCB 34991); Três Barras do Paraná, rio Adelaide, 5/IX/1997, Souza et al. s.n. (UPCB 34963); 4/X/1997, (UPCB 34978); 7/X/1997, (UPCB 34986); 6/II/1998, (UPCB 35038); Três Barras do Paraná, rio Tormenta, 5/V/1997, Souza et al. s.n. (UPCB 34911); 2/VI/1997, (UPCB 34924); 11/VII/1997, (UPCB 34952); Três Barras do Paraná, rio Guarani, 9/III/1997, Souza et al. s.n. (UPCB 34887); 6/VII/1997, (UPCB 34940); Salto do Lontra, rio Jaracatiá, 8/IV/1997, Souza et al. s.n. (UPCB
34903); 8/VII/1997, (UPCB 34957); Barra do Chopim, rio Iguaçu, 12/III/1997, Souza et al. s.n. (UPCB 34891); 9/VII/1997, (UPCB 34959).

2. Diploneis pseudovalis Hustedt var. pseudovalis, in Pascher, Süssw. -Fl. Mitteleur. 10(2): 253, fig. 403. 1930.

Fig. 16.

Valvas largamente elípticas; extremidades arredondadas; área axial estreita; área central circular; rafe reta incluída em canal longitudinal cuja ornamentação é composta por fileira simples de aréolas, distinto do restante da valva; estrias radiadas; formadas por fileira dupla de aréolas paralelas. Eixo apical: 24 a 34,4 $\mu \mathrm{m}$; eixo transapical: 16 a 18,4 $\mu \mathrm{m}$; 9 a 14 estrias em $10 \mu \mathrm{m}$.

Diploneis pseudovalis Hustedt é morfologicamente semelhante a $D$. subovalis Cleve, do qual se diferencia pela disposição paralela das aréolas nas fileiras entre duas costelas. As medidas de eixo transapical apresentadas por Patrick \& Reimer (1966) Krammer \& Lange-Bertalot (1986) foram inferiores (de 9 a $14 \mu \mathrm{m}$ ) às encontradas no material estudado. Entretanto, a disposição das aréolas é característica determinante para a identificação desta espécie.

Entre as espécies de Diploneis com fileira dupla de aréolas entre costelas, Krammer \& Lange-Bertalot (1986) distinguiram D. pseudovalis Hustedt de D. subovalis Cleve e D. parma Cleve pela disposição das aréolas nas fileiras, que estão dispostas em paralelo na primeira, e em qüincunce nas duas últimas espécies citadas.

Distribuição no Estado do Paraná: Cascavel (Tavares \& Valente-Moreira 2000).

Material examinado: BRASIL. Paraná: Capitão Leônidas Marques, rio Iguaçu, 2/IX/1997, Souza et al. s.n. (UPCB 34960); Três Barras do Paraná, rio Tormenta, 1/XII/1997, Souza et al. s.n. (UPCB 35010); Salto do Lontra, rio Jaracatiá, 7/XII/1997, Souza et al. s.n. (UPCB 35015). 
3. Diploneis subovalis Cleve var. subovalis, Syn. Navic. Dist. Kong. p. 96, pl. 1, fig. 7. 1894.

Fig. 11-15.

Valvas elípticas; extremidades arredondadas; área axial estreita; área central circular; rafe reta incluída em canal longitudinal cuja ornamentação é composta por mais de uma fileira de aréolas, distinto do restante da valva; estrias transapicais radiadas ao longo da valva; fileira dupla de aréolas dispostas em qüincunce. Eixo apical: 16 a $36 \mu$ m; eixo transapical: 8,8 a 18,4 $\mu$ m; 9 a 14 estrias em $10 \mu \mathrm{m}$.

Segundo Jensen (1985), D. subovalis Cleve pode apresentar uma fileira única ou dupla de aréolas, uma vez que fileiras simples podem transformar-se em fileiras duplas e vice-versa no mesmo indivíduo. O autor citou ainda que um estudo em formas menores de D. subovalis Cleve mostrou que estas apresentam fileiras únicas de aréolas e talvez por isso sua identificação seja tão problemática. Gandhi (1966) comentou que, em decorrência da redução do tamanho das costelas, a visão (sob microscopia ótica) de fileiras duplas ou simples de aréolas em $D$. subovalis Cleve varia de acordo com o foco que é dado no material.

Os exemplares examinados no presente trabalho variaram significativamente em tamanho, porém não morfologicamente, coincidindo com o material apresentado por Cleve-Euler (1953), Jensen (1985) e Krammer \& Lange-Bertalot (1986).

Distribuição no Estado do Paraná: Maringá (Rodrigues 1991); Ponta Grossa (Moro \& Fürstenberger 1993; Moro et al. 1994).

Material examinado: BRASIL. Paraná: Capitão Leônidas Marques, rio Iguaçu, 1/X/1997, Souza et al. s.n. (UPCB 34975); Nova Prata do Iguaçu, rio Cotegipe, 7/I/98, Souza et al. s.n. (UPCB 35021); Três Barras do Paraná, rio Adelaide, 10/I/1998, Souza et al. s.n. (UPCB 35023); Três Barras do Paraná, rio Tormenta, 11/VII/1997, Souza et al. s.n. (UPCB 34952); 1/X/1997, (UPCB 34980); 7/I/1998, (UPCB 35025); Três Barras do Paraná, rio Guarani, 5/VII/1997, Souza et al. s.n. (UPCB 34955); 12/I/1998, (UPCB 35028); Barra do Chopim, rio Chopim, 8/VII/1997, Souza et al. s.n. (UPCB 34958); 7/X/1997, (UPCB 34986); Barra do Chopim, rio Iguaçu, 9/X/1997, Souza et al. s.n. (UPCB 34987).

\section{Diploneis sp.}

Fig. 19.

Valva largamente elíptica; extremidades largamente atenuado-arredondadas; área axial estreita; área
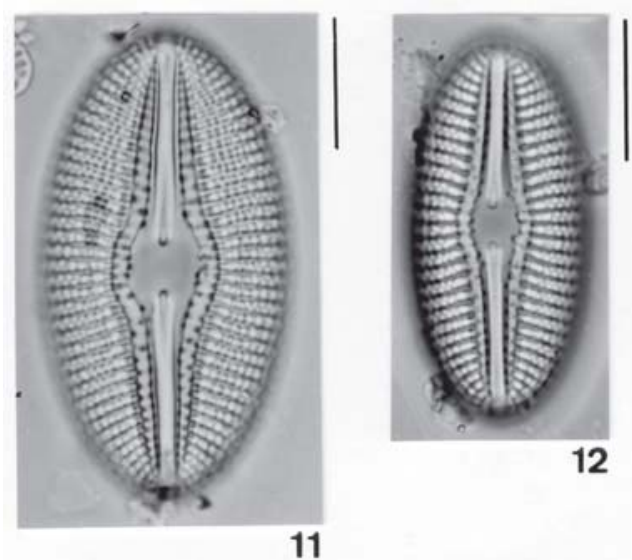

12

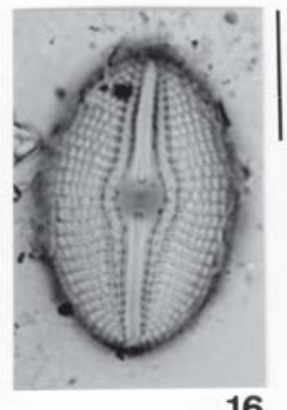

16

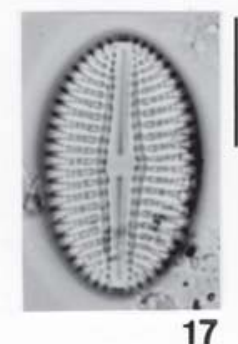

17
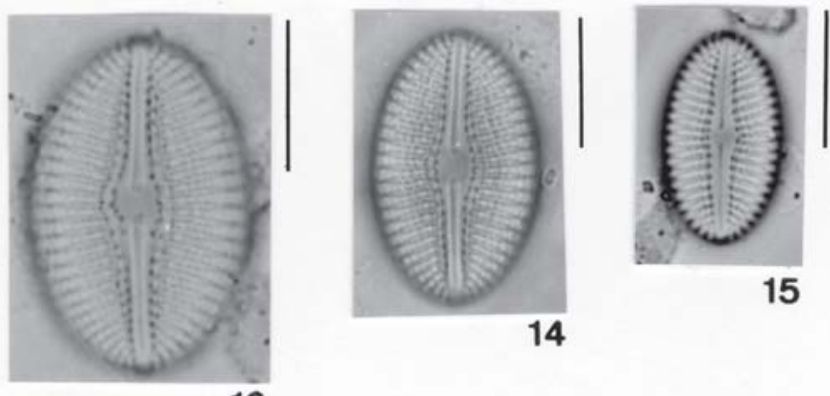

13
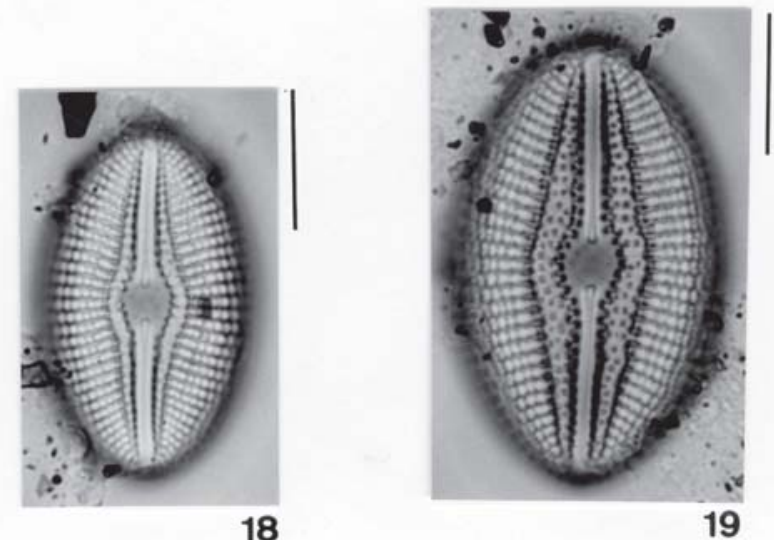

Figuras 11-19. Diploneidaceae da bacia do rio Iguaçu, PR, Brasil. 11-15. Diploneis subovalis Cleve var. subovalis. 16. Diploneis pseudovalis Hustedt var. pseudovalis. 17-18. Diploneis ovalis (Hilse) Cleve var. ovalis. 19. Diploneis sp. 
central fortemente circular; rafe reta incluída em canal longitudinal cuja ornamentação é composta por aréolas grosseiras, transversalmente uma a três aréolas na continuidade das estrias, em fileira simples, longitudinalmente, mais de uma fileira de aréolas; estrias radiadas; formadas por fileira dupla de aréolas paralelas. Eixo apical: 34,4 $\mu \mathrm{m}$; eixo transapical: 21,6 $\mu \mathrm{m} ; 12$ estrias em $10 \mu \mathrm{m}$.

O exemplar observado mostra semelhança com $D$. finnica Cleve, principalmente, pela largura do canal longitudinal (1/3 do eixo transapical). No entanto, analisando-se os espécimes estudados por Idei \& Kobayasi (1989), D. finnica apresenta aberturas quadrangulares marginais, as quais são estruturas diferenciadas das demais aréolas e podem ser visualizadas ao longo das estrias. Além disso, o canal longitudinal encontra-se ornamentado por aréolas delicadas e em fileira dupla, mais numerosas do que as observadas em exemplar estudado. As aréolas presentes nas estrias estão dispostas em qüincunce em Diploneis finnica e não paralelas, como em Diploneis sp. Portanto, optou-se por não se identificar este táxon em nível específico, principalmente porque apenas um exemplar foi observado. O mesmo apresenta semelhança no formato da valva com D. elliptica Cleve, porém o último possui fileira única de aréolas entre as costelas.

Material examinado: BRASIL. Paraná: de Barra do Chopim, rio Chopim, 7/X/1997 Souza et al. s.n. (UPCB 34986).

\section{Agradecimentos}

À equipe de campo da Companhia Paranaense de Energia, principalmente ao Sr. Gustavo Alves de Souza, pelas coletas de amostras; ao Departamento de Genética da Universidade Federal do Paraná, pelo uso da sala de revelação fotográfica; ao Conselho Nacional de Desenvolvimento Científico e Tecnológico, pela bolsa de Mestrado concedida ao primeiro autor.

\section{Referências bibliográficas}

Bittencourt-Oliveira, M.C. 2002. A comunidade fitoplanctônica do rio Tibagi: uma abordagem preliminar de sua diversidade. Pp. 373-402. In: M.E. Medri (ed.). A bacia do rio Tibagi, Londrina, M.E. Medri.

Brassac, N.M.; Atab, D.R.; Landucci, M.; Visinoni, N.D. \& Ludwig, T.A.V. 1999. Diatomáceas cêntricas de rios na região de abrangência da Usina Hidrelétrica de Salto Caxias, PR (Bacia do Rio Iguaçu). Acta Botanica Brasilica 13(3): 277-289.
Cecy, I.I.T. 1986. Estudo das algas microscópicas (Nostocophyta, Euglenophyta, Chrysophyta e Chlorophyta) do Lago do Parque Barigüi, em Curitiba, estado do Paraná, Brasil. Arquivos de Biologia e Tecnologia 29(2): 383-405.

Cleve-Euler, A. 1952. Die diatomeen von Scheweden und Finnland. Kunglika Svenska Vetenskapsakademiens Handligar 3(3): 1-153.

Cleve-Euler, A. 1953. Die diatomeen von Scheweden und Finnland. Kunglika Svenska Vetenskapsakademiens Handligar 4(5): 1-225.

Contin, L.F. 1990. Contribuição ao estudo das diatomáceas (Chrysophyta, Bacillariophyceae) na região de captação d’água do rio Iguaçu (SANEPAR), em Curitiba, estado do Paraná, Brasil. Estudos de Biologia (24): 5-95.

Costa, J.C.F. 1995. Diatomáceas (Bacillariophyceae) da reserva biológica Poço das Antas, município de Silva Jardim, Rio de Janeiro, Brasil. Iheringia série Botânica (41): 47-81.

Frengueli, J. 1923. Contribuciones para la sinopsis de las diatomeas argentinas. I. Diatomeas del rio Primeiro en la ciudad de Córdoba. Boletín da Academia Nacional de Ciencias de Córdoba 18: 13-119.

Gandhi, H.P. 1966. The freshwater diatomoflora of the JogFalls, Mysore State. Nova Hedwigia 11(1/4): 89-197.

Germain, H. 1981. Flore des Diatomées. Paris, Société Nouvelle des Éditions Boubée.

Hustedt, F. 1930. Bacillariophyta (diatomeae). In A. Pascher. Die Süsswasser flora Mittleleuropas. Jena, G. Fischer, v.10.

Idei, M. \& Kobayasi, H. 1989. The fine structure of Diploneis finnica with special reference to the marginal openings. Diatom Research 4(1): 25-37.

Intertechne. 1993. U.H. Salto Caxias, Rio Iguaçu, PR: Relatório de impacto ambiental. Curitiba, Companhia Paranaense de Energia.

Jensen, N.C. 1985. Hustedt's “Die Kieselalgen, 2. Teil”: The Pennate Diatoms. Koenigstein, Koeltz Scientific Books.

Krammer, K. \& Lange-Bertalot, H. Bacillariophyceae : Naviculaceae. 1986. In: H. Etll; I. Gerloff; H. Heynig \& D. Mollenhauer. Süsswasser flora von Mittleleuropa. G. Fischer, Sttuttgart. v.2; p. 1; 876 p.

Lange-Bertalot, H. 1980. Zur systematischen Bewertung der bandförmigen Kolonien bei Navicula und Fragilaria. Nova Hedwigia 33: 723-787.

Leandrini, J.A.; Moreira Filho, H. \& Rodrigues, L. 2002. Espécies perifíticas de Navicula Bory de dois sistemas lóticos do município de Maringá, estado do Paraná, Brasil. Hoehnea 29(1): 49-56.

Lozovei, A.L. \& Shirata, M.T. 1990. Diatomáceas (Chrysophyta, Bacillariophyceae) no rio Passaúna, Curitiba, Paraná, Brasil: levantamento qualitativo da diatomoflórula em segmento de manancial. Estudos de Biologia (27): 5-56.

Ludwig, T.A.V. \& Flôres, T. 1995. Diatomoflórula dos rios da região a ser inundada para a construção da Usina Hidrelétrica de Segredo, Paraná; I. Coscinodiscaceae, Bacillariophyceae (Achnanthales e Eunotiales) e Fragilariophyceae (Meridion e Asterionella). Arquivos de Biologia e Tecnologia 38(2): 631-650. 
Ludwig, T.A.V. \& Flôres, T. 1997. Diatomoflórula dos rios da região a ser inundada para a construção da Usina Hidrelétrica de Segredo, Paraná: Fragilariophyceae (Fragilaria e Synedra). Hoehnea 24(1): 55-65.

Metzeltin, D. \& Lange-Bertalot, H. 1998. Tropical Diatoms of the South America I. Iconographia Diatomologica 5: 1-695.

Moreira Filho, H. \& Valente-Moreira, I.M. 1981. Avaliação taxonômica e ecológica das diatomáceas (Bacillariophyceae) epífitas em algas pluricelulares obtidas nos litorais dos estados do Paraná, Santa Catarina e São Paulo. Boletim do Museu Botânico Municipal (47): 1-17.

Moro, R.S. \& Fürstenberger, C.B. 1993. Diatomáceas (Bacillariophyceae) da Lagoa Dourada (Parque Estadual de Vila Velha), Paraná, Brasil. Acta Biológica Paranaense 22(1/4): 15-30.

Moro, R.S.; Garcia, E. \& Oliveira Júnior, H.F. 1994. Diatomáceas (Bacillariophyceae) da represa Alagados, Ponta Grossa, Brasil (exclusive cêntricas). Iheringia série Botânica (45): 5-19.

Moser, G.; Steindorf, A. \& Lange-Bertalot, H. 1995. Neukaledonien Diatomeenflora einer Tropeninsel. Revision der Collection Maillard und Untersuchung neuen Materials. Bibliotheca Diatomologica 32: 1-340.
Patrick, R. \& Reimer, C.W. 1966. The diatoms of United States. Monographs of the Academy of Natural Sciences of Philadelphia 1: 1-688.

Rodrigues, L. 1991. Naviculaceae (Bacillariophyceae) nas Lagoas do Horto Florestal Dr. Luiz Teixeira Mendes, Município de Maringá, Paraná, Brasil. Revista Unimar 13(2): 273-298.

Round, F.E.; Crawford, R.M. \& Mann, D.G. 1990. The diatoms - biology and morphology of the genera. Cambridge, Cambridge University Press.

Suderhsa. 1995. Qualidade das Águas Interiores do Estado do Paraná: 1987-1995. Curitiba, Superintendência de Desenvolvimento de Recursos Hídricos e Saneamento Ambiental.

Tavares, B. \& Valente-Moreira, I.M. 2000. Diatomoflórula do lago de Cascavel, município de cascavel, estado do Paraná, Brasil. Hoehnea 27(1): 1-24.

Valente-Moreira, I.M. 1975. Contribuição ao estudo das Bacillariophyceae (Diatomáceas em diatomitos brasileiros). Acta Biológica Paranaense 4(3/4): 135-198.

Van Heurck, H. 1880-85. Synopsis des diatomées de Belgique. Anver, L’Auteur. 